\title{
Can circumcision be avoided in adult male with phimosis? Results of the PhimoStop ${ }^{\mathrm{TM}}$ prospective trial
}

\author{
Marco Carilli ${ }^{1 \#}$, Anastasios D. Asimakopoulos ${ }^{1 \#}$, Serena Pastore ${ }^{1}$, Stefano Germani ${ }^{1}$, Luca Orecchia ${ }^{1}$, \\ Enrico Finazzi Agrò ${ }^{2}$, Roberto Miano ${ }^{2}$ \\ ${ }^{1}$ Division of Urology, Fondazione PTV Policlinico Tor Vergata, Rome, Italy; ${ }^{2}$ Department of Surgical Sciences, Division of Urology, University of \\ Rome Tor Vergata, Rome, Italy \\ Contributions: (I) Conception and design: R Miano, AD Asimakopoulos; (II) Administrative support: E Finazzi Agrò; (III) Provision of study materials \\ or patients: R Miano, AD Asimakopoulos; (IV) Collection and assembly of data: M Carilli, S Pastore; (V) Data analysis and interpretation: M Carilli, \\ R Miano; (VI) Manuscript writing: All authors; (VII) Final approval of manuscript: All authors. \\ \#These authors contributed equally to this work and considered as first authors. \\ Correspondence to: Dr. Marco Carilli, MD. Division of Urology, Fondazione PTV Policlinico Tor Vergata, Rome, Italy. \\ Email: carillimarco@gmail.com.
}

Background: Circumcision as surgical treatment of adult phimosis is not devoid of complications. Efficacy of alternative non-surgical options is unclear. PhimoStop ${ }^{\text {TM }}$ is a therapeutic protocol which involves the use of appropriately shaped silicone tuboids of increasing size to obtain a non-forced dilation of the prepuce. The aim of the study was to evaluate the efficacy and durability of results of PhimoStop ${ }^{\mathrm{TM}}$ device for the treatment of adult male phimosis.

Methods: A prospective trial was conducted between 2018 and 2020 on 85 consecutive adult male patients affected by phimosis and with an indication for circumcision. Patients were treated with PhimoStop ${ }^{\text {TM }}$ protocol and they were evaluated at baseline and after treatment through a subjective (patient selfreported information on various domains of his sexual function) and an objective assessment (evaluation of phimosis severity grade according to the Kikiros scale pre- and post-treatment, re-assessment of indication for circumcision post-treatment and validated questionnaires scores). Primary endpoint was to avoid the scheduled circumcision in $33 \%$ of the patients enrolled.

Results: Seventy-one patients (84\%) completed the device usage phase as per study protocol. Median duration of tuboid application was 60 days. Thirty-seven patients $(52.1 \%)$ had no indication for circumcision after treatment. Even considering patients lost to follow-up as failures, primary endpoint was reached in $43.5 \%$ of cases. There was a significant reduction of the grade of phimosis after treatment $(\mathrm{P}<0.001)$. Moreover IIEF-5 showed a statistically significant improvement after treatment $(\mathrm{P}<0.001)$. Thirty/37 patients who met the primary endpoint $(81 \%)$ still have a successful resolution of their phimosis avoiding circumcision at a median follow-up of 24 months.

Conclusions: PhimoStop ${ }^{\text {TM }}$ device is effective for the treatment of adult male phimosis of Kikiros grade $\leq 2$. The results seem to be durable in most patients at a median follow-up of 24 months. Randomized clinical trials are necessary in order to confirm our results and assess cost-efficacy.

Keywords: Phimosis; circumcision; non-surgical treatment of phimosis; Kikiros grade; adult male phimosis

Submitted Jul 30, 2021. Accepted for publication Oct 04, 2021.

doi: $10.21037 /$ tau-21-673

View this article at: https://dx.doi.org/10.21037/tau-21-673 


\section{Introduction}

Phimosis is defined as the inability to retract the foreskin or prepuce behind the glans penis. Pathologic phimosis is the adult form of the disease as a result of local scarring, infection or inflammation due to poor hygiene, recurrent balanitis and/or posthitis, diabetes mellitus and balanitis xerotica obliterans (BXO). Forceful foreskin retraction can lead to fissures, bleeding, scarring and consequent worsening of the condition (1).

Treatment of adult phimosis varies depending on the age of the patient and severity of the condition. Traditionally, surgical treatment through circumcision under local anaesthesia is considered the gold standard treatment of adult male phimosis and several surgical techniques have been described (2). Usually, circumcision is a simple and safe surgical procedure. Nevertheless, is not devoid of complications, with an overall complication risk of $3.8 \%$ (3). Bleeding, pain, wound infection, concealed penis, skin bridge, urinary retention, recurrent phimosis, fistulas, necrosis, iatrogenic hypospadias and epispadias, meatitis, meatal stenosis, chordee, redundant skin, non-satisfying cosmetic appearance and impotence are the described complications, most of which may significantly impact on healthcare costs $(4,5)$.

Several therapeutic options alternative to or assisting conventional circumcision have been described, these often involve the application of several in-situ devices which generally aim to crush the foreskin while simultaneously creating hemostasis; the foreskin is then excised or allowed to slough off. Use of circumcision devices requires local anaesthesia and may significantly reduce operative times at the risk of an increase of moderate adverse effects (6-8).

Non-surgical treatment of adult phimosis is principally based on the application of topical corticosteroid ointments of different potency and concentration, for 4-8 weeks. Compared to placebo or manual reduction, corticosteroids significantly increase complete or partial clinical resolution of phimosis; however, no long-term follow-up data have been reported on the durability of the results $(9,10)$.

In clinical practice, is not unusual that patients refuse circumcision: in fact, postoperative care can be bothersome, and not all patients accept the impact of surgery on genital self-image. In some cases, circumcision could result in "overtreatment", especially in mild phimosis.

Hypothesis is that the use of a non-surgical treatment protocol, such as the novel minimally invasive device PhimoStop ${ }^{\mathrm{TM}}$, could avoid circumcision in a significant number of patients with mild-moderate phimosis. Objective of the study was to prospectively assess the efficacy and durability of results of PhimoStop ${ }^{\mathrm{TM}}$.

We present the following article in accordance with the TREND reporting checklist (available at https://dx.doi. org/10.21037/tau-21-673).

\section{Methods}

PhimoStop ${ }^{T M}$ device

PhimoStop ${ }^{\text {TM }}$ (Phimomed S.r.1., Rome, Italy) is a therapeutic protocol which involves the use of appropriately shaped silicone tuboids of increasing size (Figure 1A). The device has been validated by Italian Ministry of Health on 13/01/2014 (No. 1058196), and it obtained an EU patent (No. 2648642) with CE mark.

Tuboids are shaped to be ergonomic when applied on the glans. Once the foreskin has been fully retracted, the tuboid can be applied on the glans with the central hole overlaid on the urethral meatus, allowing the patient to urinate without removing the device; the inner foreskin is then pulled back alongside the lateral aspect of the tuboid. In this way, the phimotic ring lies on the cylindrical portion of the tuboid, thus undergoing a slightly forced and progressive dilation. The fins of the tuboids are lowered on the prepuce and fixed in place with adhesive patches.

The standard progressive tuboids are available in seven different sizes, from 1 (diameter: $6 \mathrm{~mm}$ ) to 7 (diameter: $35 \mathrm{~mm}$ ). The package also includes some intermediate rings that, when placed on the standard ones, allow a more gradual increase of the tuboid diameter of half a size, from $2.5(12.5 \mathrm{~mm})$ to $7.5(37.5 \mathrm{~mm})$, which is the maximum available tuboid diameter (Figure $1 B$ ). Intermediate rings are designed to avoid a steep and possibly painful stretching of the prepuce.

\section{Ethics}

The study was conducted in accordance with the Declaration of Helsinki (as revised in 2013). The study was approved by institutional ethics committee of Policlinico Tor Vergata Foundation (protocol No. 231/16) and informed consent was taken from all the patients

\section{Patients}

Between January 2018 and January 2020, 85 consecutive 

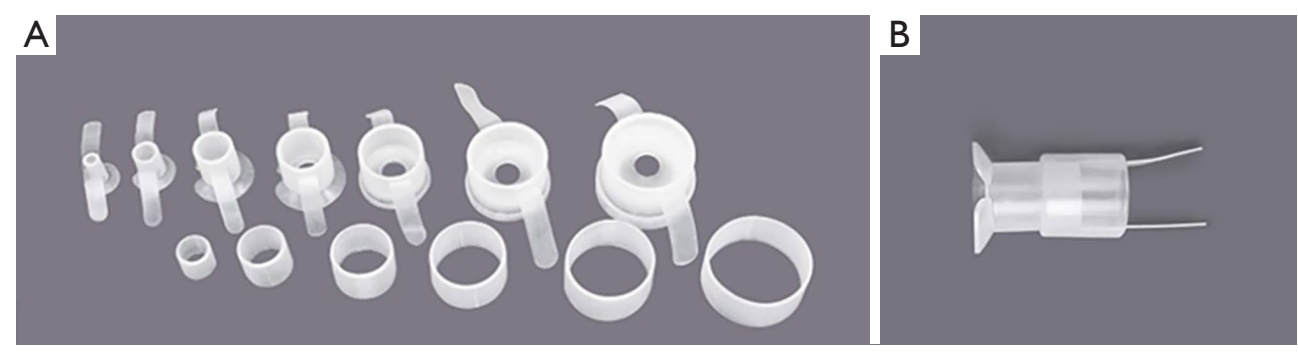

Figure 1 PhimoStop ${ }^{\text {TM }}$ device. (A) Standard tuboids (top row) and intermediate tuboids (bottom row). The fins of standard tuboids are fixed outside the prepuce with adhesive patches. (B) Insertion of the intermediate tuboid on the standard one, allowing a gradual increase of the tuboid diameter of half a size.

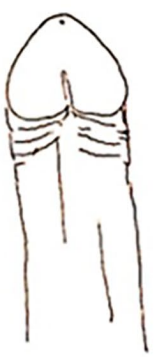

0

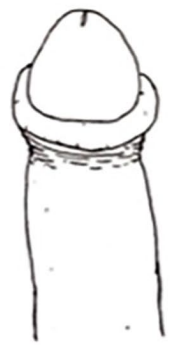

1

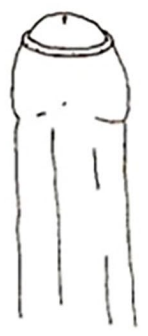

2

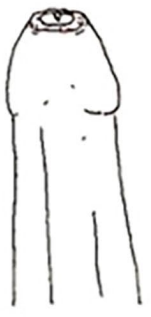

3

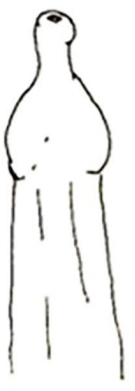

4-5

Figure 2 Kikiros classification of phimosis severity grade: grade 0 = full retractability; grade 1 = full retraction but tight behind glans; grade 2 = partial exposure of glans; grade 3 = partial retraction, meatus just visible; grade 4 = slight retraction, but some distance between tip and glans, i.e., neither meatus nor glans can be exposed; grade $5=$ absolutely no retraction.

adult male patients affected by phimosis and with an indication for circumcision were enrolled in a prospective, single centre and non-profit study.

Patients were enrolled if they had a phimosis severity grade $\leq 2$ according to the Kikiros scale (grade 0: full retractability; grade 1: full retraction but tight behind glans; grade 2: partial exposure of glans) (Figure 2) (11). Exclusion criteria are reported in Table 1. Patients with phimosis grade $>2$ were excluded because of the impossibility to retract the prepuce sufficiently to apply PhimoStop ${ }^{\mathrm{TM}}$. Patients with full retraction of the foreskin and evidence of phimosis only during the erectile phase were classified as grade 0 .

Data was registered into an anonymised database; each patient was assigned a unique study code.

\section{Study end-point and definition of failure}

Primary endpoint was to avoid the scheduled circumcision in $33 \%$ of the patients enrolled after using the PhimoStop ${ }^{\mathrm{TM}}$ device. Success was then defined as the avoidance of the scheduled circumcision based on both subjective and objective parameters.

Failure was defined as the persistence of the indication for surgical treatment.

\section{Device usage protocol}

Based on phimosis severity and penile conformation, the smaller tuboid size patients were able to wear was initially applied. As the phimotic ring expanded, patients were trained to progressively increase tuboid size, using also the intermediate tuboids when necessary to avoid excessive prepuce forcing and pain. Whenever pain with the a new tuboid size occurred, patients were instructed to return to the previous one and reapply it for a longer time.

It was suggested to use each tuboid for $48-72$ hours before increasing size; however, the patient was free to decide the timing of moving to the next tuboid. Device application could be eased by topical emollient or lubricating ointment, if necessary. The active dilation 
Table 1 Exclusion criteria

Absence of informed consent

Kikiros grade $3-4-5$

Active or suspected uro-genital infections (including sexually-transmitted infections)

Any of the following: genital ulcers; paraphimosis; hypospadias; epispadias; clinical balanitis xerotica obliterans (BXO); uncontrolled diabetes

Patients unfit for device use (e.g., compromised neurological status, low compliance)

Known or suspected hypersensitivity to tuboid material

process ended when the maximum size of tuboids was reached in relation to each individual prepuce. At that time foreskin retraction alongside the glans was tested by the patient during flaccid and erected phase. During the following weeks (maintenance phase), the use of the tuboids was left to individual will. Device hygiene was maintained during the study period through cleaning with water and soap.

\section{Main outcome measures}

Patients were evaluated at baseline and after 4 months from enrollment through a subjective and an objective assessment. Every patient provided information on the duration of the treatment and the maximum tuboid size used at the start and the end of treatment. All causes of discontinuation of therapy and side-effects were recorded. The subjective assessment aimed to gather patient self-reported information on various domains of his sexual function (penile sensitivity, erection, ejaculation, orgasm, pain during sexual intercourse). The objective assessment was performed by two dedicated urologists (M.C.; S.P.) through the evaluation of the Kikiros grade of phimosis pre- and post-treatment, re-assessment of indication for circumcision post-treatment and using validated questionnaires: Male Genital Self-Image Scale (MGSIS-7) (12), Erection Hardness Score (EHS) (13) and International Index of Erectile Function (IIEF-5) (14).

\section{Assessment of durability of results}

On December 2020, patients who met the primary endpoint received a telephone interview performed by a third party (physician non directly involved to the patient care). The interview consisted of two questions: (I) "Was there any need to reuse the device after completion of the study?" and
(II) "Did you undergo circumcision after completion of the study?". All responses were recorded and correlated with the baseline data, in particular Kikiros grade at baseline and after PhimoStop ${ }^{\mathrm{TM}}$ treatment.

\section{Statistical analysis}

Based on the assumption that "success" was defined as the avoidance of at least $33 \%$ of the scheduled circumcisions, a sample size of at least 85 patients was required to provide a $95 \%$ confidence interval and a $10 \%$ error. Chi-square and Fisher's exact tests were used for categorical variables as appropriate. Student's $t$ test for unpaired data or MannWhitney test were used to compare variations of Kikiros grade and questionnaires scores before and after treatment. Primary endpoint was calculated both on the entire enrolled population (FAS-full analysis set-population) and among patients with complete follow-up data (PP-per protocolpopulation). In FAS analysis, missing data of patients lost to follow-up were considered as "worst case", i.e., assuming that they were scheduled for circumcision. The percentage of patients who avoided circumcision and the corresponding Clopper-Pearson exact confidence interval have been reported. Data analysis was conducted using Stata 16.1 software (StataCorp LLC, College Station, TX, USA). Statistical significance was defined as $\mathrm{P}$ value $<0.05$.

\section{Results}

A total of 85 patients were enrolled and their clinical and demographic baseline characteristics are summarized in Table 2. Fourteen patients were lost to follow-up (16\%) and did not complete the device usage phase as per study protocol; comparison of baseline data between patients with complete follow-up and patients lost to follow-up did not show any statistically significant differences. 
Table 2 Clinical and demographic characteristics

\begin{tabular}{|c|c|c|c|c|}
\hline Characteristics & Total $(\mathrm{N}=85)$ & Patients with complete follow-up ( $\mathrm{N}=71$ ) & Patients lost to follow-up ( $\mathrm{N}=14)$ & $P$ value \\
\hline Mean \pm SD & $33.8 \pm 15.1$ & $34.3 \pm 15.6$ & $30.6 \pm 12.1$ & 0.631 \\
\hline Median (Q1-Q3) & $30(21.0-45.0)$ & $32(20.0-45.5)$ & $27(22.8-32.8)$ & \\
\hline Min-Max & $18-69$ & $18-69$ & $18-59$ & \\
\hline Diabetes & 8 & 7 & 1 & \\
\hline Hypertension & 7 & 6 & 1 & \\
\hline Dyslipidemia & 7 & 6 & 1 & \\
\hline Other & 16 & 14 & 2 & \\
\hline Kikiros 1 & $37(43.5)$ & $29(40.8)$ & $8(57.1)$ & \\
\hline Kikiros 2 & $16(18.8)$ & $14(19.7)$ & $2(14.3)$ & \\
\hline
\end{tabular}

$\wedge$, calculated on patients with at least one comorbidity. ${ }^{*}$, other comorbidities included: benign prostatic hyperplasia (4 cases); hypothyroidism (4 cases); asthma (3 cases); hyperuricemia (2 cases); depression (1 case); Behçet's syndrome (1 case); retinitis pigmentosa (1 case). SD, standard deviation; Q1-Q3, interquartile range.

Table 3 Duration of treatment, initial and maximum tuboid size

\begin{tabular}{lc}
\hline Variable & Value \\
\hline Duration of treatment (days) & $62.7 \pm 31.6$ \\
Mean \pm SD & $60[35-90]$ \\
Median [Q1-Q3] & $10-120$ \\
Min-Max & \\
Maximum tuboid size & $4.97 \pm 1.1$ \\
Mean \pm SD & $5[4-6]$ \\
Median [Q1-Q3] & $2-7$ \\
Min-Max & \\
Initial tuboid size & $3.12 \pm 0.7$ \\
Mean \pm SD & $3[3-3.5]$ \\
Median [Q1-Q3] & $1-6$ \\
Min-Max &
\end{tabular}

$\mathrm{SD}$, standard deviation; Q1-Q3, interquartile range.

Mean initial tuboid size applied and mean maximum tuboid size used was $3.12 \pm 0.7$ and $4.96 \pm 1.1$ respectively. Median duration of tuboid application reported by patients was 60 days, as reported in Table 3.
Among PP, 37 patients (52.1\%; 95\% CI: 39.9-64.1\%) had no indication for circumcision after treatment. 7/37 (19\%) had residual phimosis, all classified as Kikiros grade 0 (6/7 had Kikiros grade 1 and 1/7 grade 2 pre-treatment), but were completely satisfied from the treatment and considered surgical treatment as not useful for them. 34 patients $(47.9 \%)$ had persistent phimosis at final evaluation with confirmation for circumcision.

Even considering patients lost to follow-up as failures ( $\mathrm{N}=85$; “worst case" scenario), primary endpoint was reached (43.5\%; 95\% CI: 33-55\%).

A total of $57 / 71$ patients $(80 \%)$ would recommend PhimoStop ${ }^{\mathrm{TM}}$ to other patients with phimosis and a total of $58 \%$ of patients $(41 / 71)$ were satisfied with the results obtained (Table S1).

Focusing on Kikiros grade variation, there was a significant downgrading after treatment $(\mathrm{P}<0.001)$. Moreover, IIEF-5 score showed a statistically significant improvement after treatment $(\mathrm{P}<0.001)$, while MGSIS-7 and EHS scores did not show any statistically significant differences compared to the baseline. Tables 4 and 5 show the results.

Thirty patients who completed the follow-up visits, referred to have spontaneously discontinued the treatment 
Table 4 Kikiros grade comparison before and after treatment (PP)

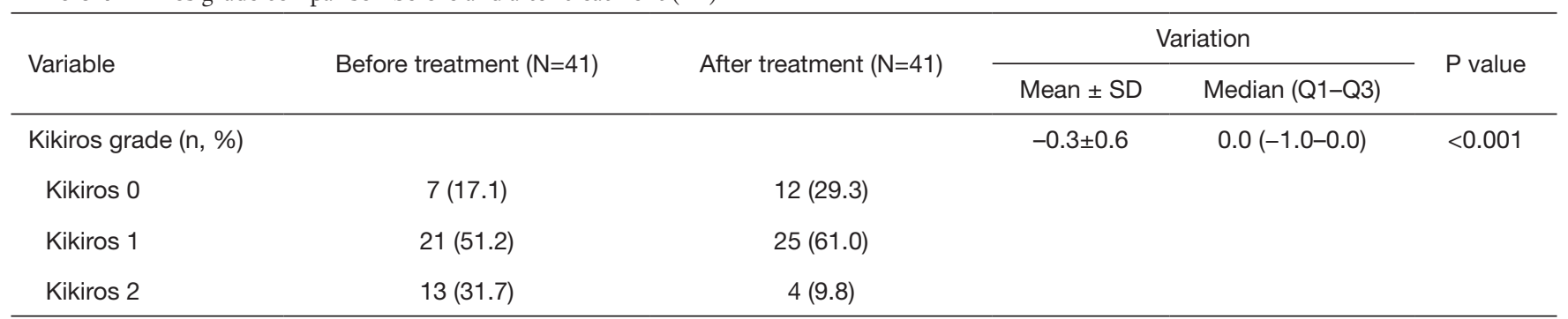

SD, standard deviation; Q1-Q3, interquartile range.

Table 5 Questionnaires score comparison before and after treatment (PP)

\begin{tabular}{|c|c|c|c|c|c|}
\hline Variable & Before treatment $(\mathrm{N}=71)$ & After treatment $(\mathrm{N}=71)$ & \multicolumn{2}{|c|}{ Variation } & $P$ value \\
\hline MGSIS-7 score & & & $0.2 \pm 2.6$ & $0(0-1)$ & 0.467 \\
\hline Mean \pm SD & $21.7 \pm 3.3$ & $21.5 \pm 3.3$ & & & \\
\hline Median (Q1-Q3) & $21(20-22)$ & $21(20-23)$ & & & \\
\hline Mean \pm SD & $16.3 \pm 7.3$ & $17.5 \pm 7.3$ & & & \\
\hline Median (Q1-Q3) & $19(10.5-22.5)$ & $21(12-23)$ & & & \\
\hline EHS score (n, \%) & & & $-0.06 \pm 0.4$ & $0(0-0)$ & 0.388 \\
\hline Score 2 & $1(1.4)$ & $2(2.8)$ & & & \\
\hline
\end{tabular}

MGSIS-7, Male Genital Self-Image Scale; IIEF-5, International Index of Erectile Function; EHS, Erection Hardness Score; SD, standard deviation; Q1-Q3, interquartile range.

Table 6 Discontinuation of treatment and side-effects

\begin{tabular}{lc}
\hline Variable & Value \\
\hline Not satisfied & $9(30 \%)$ \\
Adoption of other therapeutic options & \\
Local ointment & $1(3.3 \%)$ \\
Circumcision & $3(10 \%)$ \\
Difficult use & $1(3.3 \%)$ \\
Foreskin superficial lesions & $3(10 \%)$ \\
Discomfort with large tuboid size & \\
Tuboid size between 3 and 5 & $8(26.7 \%)$ \\
Tuboid size $>5$ & $5(16.7 \%)$ \\
Not satisfied & $9(30 \%)$ \\
\hline
\end{tabular}

before the final urologist evaluation. 26/30 (87\%) of those patients were part of the group who failed treatment, while 4/30 (13\%) simply discontinued PhimoStop ${ }^{\text {TM }}$ because of discomfort with larger tuboids not influencing the final success of the treatment. Main causes of treatment discontinuation are summarized in Table 6.

Thirty-seven patients who met the primary endpoint were interviewed on December 2020, at a median follow-up of 24.1 months after treatment (range, 12.1-35.6 months): $13 / 37$ (35\%) of patients reused the device during the follow-up phase and 24/37 (65\%) did not, with $11 / 13$ $(85 \%)$ patients that reused the device being satisfied of the obtained outcome. Two/13 (15\%) patients that reused the device and 5/24 (21\%) patients that did not reuse it decided to undergo surgery, despite being initially defined 
as "success". Consequently, 30/37 patients (81\%) still have a successful resolution of their phimosis avoiding circumcision, despite the initial indication for surgical treatment.

\section{Discussion}

Circumcision, the traditional and most used treatment of adult phimosis, usually does not lead to severe complications. However, if side effects occur, bleeding and infection are the most common (3). Concurrently, the longterm data about the use of topical corticosteroid ointments as a non-surgical therapy for adult phimosis remains unknown $(9,10)$. Several in-situ devices have been described but their use has been correlated with some drawbacks: infection, dislodgment and need of local anaesthesia (6-8).

PhimoStop $^{\mathrm{TM}}$ is a new minimally-invasive treatment for mild to intermediate adult phimosis (Kikiros grade 0-2) characterized by a good ergonomics, easiness of use, resistance to infections and no need for local anaesthesia.

Our study shows that PhimoStop ${ }^{\mathrm{TM}}$ is safe and effective as a non-surgical treatment of mild to intermediate adult phimosis. The primary endpoint was met both on the entirely enrolled population (FAS population, 43.5\%) and among patients with complete follow-up data (PP, $52.1 \%$ ) leading to the avoidance of about one half of the scheduled circumcisions. The results, achieved after a mean time of two months of therapy, have an even higher value considering that, in the current SARS-CoV-2 pandemic scenario, the healthcare systems might be overloaded and non-urgent non-oncological surgical procedure such as circumcision can be difficult to be carried out.

Among PP, most patients were satisfied after the treatment and would recommend it to other patients, although a persistent and not clinically relevant Kikiros grade 0 phimosis was observed in $19 \%$ of the patients who completed follow-up.

Regarding the subjective and objective assessment using patients self-reported information and questionnaires on various domains of sexual function, about $15 \%$ of the patients reported an improvement in erectile function and ejaculation time. The theory that phimosis resolution may help the patient to live his sexuality with less stress and anxiety leading to the improvement in erectile and ejaculatory functions was already proposed by several authors who showed the positive effect on sexual life in patients who undergone circumcision due to better erectile function and positive genital self-image $(15,16)$.
Czajkowski et al. published the results from a prospective study on 69 patients undergoing circumcision. In this study, 3 months after surgery, all patients achieved significant improvement in both obtaining and maintaining an erection based on IIEF-5 score; moreover, there was an improvement in satisfaction with genital self-image, as confirmed by MGSIS-7 score (15). In our study, only IIEF5 score showed a statistically significant improvement after treatment, while MGSIS-7 score did not show any improvement compared to the baseline. This could be explained by the fact that, unlike conventional circumcision, the use of PhimoStop ${ }^{\text {TM }}$ is not associated with "physical" removal of preputial tissue, but only with a "remodelling" of the foreskin, which could have less impact on genital selfimage. Anyway, our results show that PhimoStop ${ }^{\mathrm{TM}}$ can help patients to improve the quality of their sexual lives.

Among PP, 30 patients discontinued treatment before urological final evaluation, because of lack of satisfaction with device usage (30\%), adoption of other therapeutic options (13\%) or onset of side-effects (53\%). About $90 \%$ of those patients referred to the group of ones who failed treatment. Overall, side-effects were mild and did not lead to the interruption of the study. In most cases, they were represented by discomfort with larger tuboid size. Obviously, although tuboids have been designed to be as ergonomic as possible, subjectivity of patients' sensations, as well as inter-individual variability in penile shape and size, make the device not fit perfectly in all patients. Only one patient reported difficulties in using the device such that the treatment was suspended, proving that the device is generally easy to use after a brief training provided by the physician.

Thirty/37 (81\%) patients maintained good outcomes at a median follow-up of 24 months, confirming the durability of the results over time. This is of particular importance if we considered that long-term data on the use on nonsurgical treatment options for adult phimosis are still lacking.

Our study has some limitations. First, the study design did not include a control arm (circumcision or topical corticosteroid therapy), which makes not possible to establish a comparison with standard therapies. Second, BXO diagnosis was only clinical, since we decided to avoid any invasive procedure (e.g., penile biopsy), and thus leads to a potential discrepancy between clinical and histopathological diagnosis of BXO, as reported by Czajkowski et al. (17). Third, long-term follow up was not standardised and the modalities of the device re-use at long- 
term were not assessed (duration, tuboid size, etc.) but left to personal decision based on their previous experience: consequently, we are not able to establish whether the standardisation in device use during a second course of therapy could lead to further improvement in long-term results. Finally, cost-analysis was not part of the study and needs to be adequately assessed in the future.

\section{Conclusions}

The minimally-invasive device PhimoStop ${ }^{\mathrm{TM}}$ is effective for the treatment of adult male phimosis of Kikiros grade $\leq 2$, with more than half of patients who avoided the scheduled circumcision. The results seem to be durable in most patients at a median follow-up of 24 months. A randomized clinical trial comparing circumcision with Phimostop ${ }^{\mathrm{TM}}$ in patients with mild-to-moderate phimosis would be advisable, although the right primary endpoint in such trial would be a combination of efficacy, complications and costs.

\section{Acknowledgments}

Funding: None.

\section{Footnote}

Reporting Checklist: The authors have completed the TREND reporting checklist. Available at https://dx.doi. org/10.21037/tau-21-673

Data Sharing Statement: available at https://dx.doi. org/10.21037/tau-21-673

Peer Review File: Available at https://dx.doi.org/10.21037/ tau-21-673

Conflicts of Interest: All authors have completed the ICMJE uniform disclosure form (available at https://dx.doi. org/10.21037/tau-21-673). The authors have no conflicts of interest to declare.

Ethical Statement: The authors are accountable for all aspects of the work in ensuring that questions related to the accuracy or integrity of any part of the work are appropriately investigated and resolved. The study was conducted in accordance with the Declaration of Helsinki (as revised in 2013). The study was approved by institutional ethics committee of Policlinico Tor Vergata Foundation (protocol No. 231/16) and informed consent was taken from all the patients.

Open Access Statement: This is an Open Access article distributed in accordance with the Creative Commons Attribution-NonCommercial-NoDerivs 4.0 International License (CC BY-NC-ND 4.0), which permits the noncommercial replication and distribution of the article with the strict proviso that no changes or edits are made and the original work is properly cited (including links to both the formal publication through the relevant DOI and the license). See: https://creativecommons.org/licenses/by-nc-nd/4.0/.

\section{References}

1. Shahid SK. Phimosis in children. ISRN Urol 2012;2012:707329.

2. Holman JR, Stuessi KA. Adult circumcision. Am Fam Physician 1999;59:1514-8.

3. Shabanzadeh DM, Clausen S, Maigaard K, et al. Male Circumcision Complications - A Systematic Review, MetaAnalysis and Meta-Regression. Urology 2021;152:25-34.

4. Krill AJ, Palmer LS, Palmer JS. Complications of circumcision. ScientificWorldJournal 2011;11:2458-68.

5. Abdulwahab-Ahmed A, Mungadi IA. Techniques of male circumcision. J Surg Tech Case Rep 2013;5:1-7.

6. Hohlfeld A, Ebrahim S, Shaik MZ, et al. Circumcision devices versus standard surgical techniques in adolescent and adult male circumcisions. Cochrane Database Syst Rev 2021;3:CD012250.

7. Lebina L, Milovanovic M, Otwombe K, et al. PrePex circumcision surveillance: Adverse events and analgesia for device removal. PLoS One 2018;13:e194271.

8. Gu C, Tian F, Jia Z, et al. Introducing the Quill ${ }^{\mathrm{TM}}$ device for modified sleeve circumcision with subcutaneous suture: a retrospective study of 70 cases. Urol Int 2015;94:255-61.

9. Moreno G, Corbalán J, Peñaloza B, et al. Topical corticosteroids for treating phimosis in boys. Cochrane Database Syst Rev 2014;(9):CD008973.

10. Liu J, Yang J, Chen Y, et al. Is steroids therapy effective in treating phimosis? A meta-analysis. Int Urol Nephrol 2016;48:335-42.

11. Kikiros CS, Beasley SW, Woodward AA. The response of phimosis to local steroid application. Pediatr Surg Int 1993;8:329-32.

12. Herbenick D, Schick V, Reece M, et al. The development and validation of the Male Genital Self-Image Scale: results from a nationally representative probability sample 
of men in the United States. J Sex Med 2013;10:1516-25.

13. Mulhall JP, Goldstein I, Bushmakin AG, et al. Validation of the erection hardness score. J Sex Med 2007;4:1626-34.

14. Rosen RC, Cappelleri JC, Smith MD, et al. Development and evaluation of an abridged, 5 -item version of the International Index of Erectile Function (IIEF-5) as a diagnostic tool for erectile dysfunction. Int J Impot Res 1999;11:319-26.

15. Czajkowski M, Czajkowska K, Zarańska K, et al. Male Circumcision Due to Phimosis as the Procedure That Is Not Only Relieving Clinical Symptoms of Phimosis

Cite this article as: Carilli $\mathrm{M}$, Asimakopoulos $\mathrm{AD}$, Pastore $\mathrm{S}$, Germani S, Orecchia L, Finazzi Agrò E, Miano R. Can circumcision be avoided in adult male with phimosis? Results of the PhimoStop ${ }^{\text {TM }}$ prospective trial. Transl Androl Urol 2021;10(11):4152-4160. doi: 10.21037/tau-21-673
But Also Improves the Quality of Sexual Life. Sex Med 2021;9:100315.

16. Nordstrom MP, Westercamp N, Jaoko W, et al. Medical Male Circumcision Is Associated With Improvements in Pain During Intercourse and Sexual Satisfaction in Kenya. J Sex Med 2017;14:601-12.

17. Czajkowski M, Żawrocki A, Czajkowska K, et al. Lichen Sclerosus and Phimosis - Discrepancies Between Clinical and Pathological Diagnosis and Its Consequences. Urology 2021;148:274-9. 
Supplementary

Table S1 Endpoints in PP

\begin{tabular}{|c|c|}
\hline Variable & Value \\
\hline \multicolumn{2}{|l|}{ Patients with complete follow-up (n, \%) } \\
\hline With indication for circumcision & $34(47.9 \%)$ \\
\hline Without indication for circumcision & $37(52.1 \%)$ \\
\hline \multicolumn{2}{|l|}{ "Are you satisfied by the results?" (n, \%) } \\
\hline Yes & $41(57.7 \%)$ \\
\hline No & $25(35.2 \%)$ \\
\hline I don't know & $5(7.0 \%)$ \\
\hline \multicolumn{2}{|l|}{ "Would you recommend it?" (n, \%) } \\
\hline Yes & $57(80.3 \%)$ \\
\hline No & $9(12.7 \%)$ \\
\hline I don't know & $5(7.0 \%)$ \\
\hline \multicolumn{2}{|l|}{ Penile sensitivity (n, \%) } \\
\hline Reduced & $13(18.3 \%)$ \\
\hline Increased & $12(16.9 \%)$ \\
\hline Not modified & $46(64.8 \%)$ \\
\hline \multicolumn{2}{|l|}{ Erectile function (n, \%) } \\
\hline Improved & $10(14.1 \%)$ \\
\hline Worsened & $5(7.0 \%)$ \\
\hline Not modified & $56(78.9 \%)$ \\
\hline \multicolumn{2}{|l|}{ Ejaculation time (n, \%) } \\
\hline Improved & $9(12.7 \%)$ \\
\hline Worsened & $1(1.4 \%)$ \\
\hline Not modified & $61(85.9 \%)$ \\
\hline \multicolumn{2}{|l|}{ Orgasmic function (n, \%) } \\
\hline Improved & $1(1.4 \%)$ \\
\hline Worsened & $7(9.9 \%)$ \\
\hline Not modified & $63(88.7 \%)$ \\
\hline \multicolumn{2}{|l|}{ Persistent painful sexual intercourse (n, \%) } \\
\hline Yes & $17(23.9 \%)$ \\
\hline No & $28(39.4 \%)$ \\
\hline I don't know & $26(36.6 \%)$ \\
\hline Good cosmetic and functional result (n, \%) & $44(62.0 \%)$ \\
\hline Persistent phimosis (n, \%) & $41(57.7 \%)$ \\
\hline
\end{tabular}

